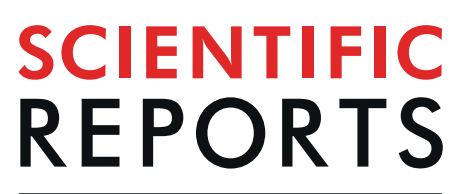

natureresearch

\title{
Essential role of the initial activation signal in isotype selection upon deletion of a transcriptionally committed promoter
}

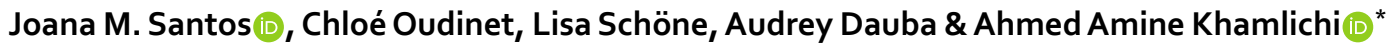

Class switch recombination (CSR), which targets exclusively the constant region of the immunoglobulin heavy chain $(I g H)$ locus, plays an important role in humoral immunity by generating different antibody effector functions. The $\mathrm{IgH}$ constant locus contains multiple genes controlled by isotype (I) promoters induced by extracellular signals that activate specific I promoters, leading to B cell commitment. However, it is unknown whether after initial commitment to one promoter, non-responsive I promoters are irreversibly silent or if they can be activated after exposure to their specific inducers. Here, we studied the murine cell line $\mathrm{CH} 12$, which is committed to produce IgA in response to TGF- $\beta$. We show that, although other promoters than l $\alpha$ are transcriptionally inactive, they are not irreversibly silent. Following deletion of the committed I $\alpha$ promoter by CRISPR/Cas9, other I promoters display a complex transcriptional pattern largely dependent on the initial committing signal.
\end{abstract}

Humoral immunity relies on a vast repertoire of B cell antigen receptors generated through different processes along B cell development ${ }^{1,2}$. At the immunoglobulin heavy chain $(\mathrm{IgH})$ locus, the variable region undergoes $\mathrm{V}(\mathrm{D})$ $\mathrm{J}$ recombination in developing B cells leading to the assembly of the variable gene segments $(\mathrm{V}(\mathrm{D}) \mathrm{J})^{3-5}$. The constant region undergoes class switch recombination (CSR), enabling IgM-expressing B cells to switch to other isotypes (IgG, IgE, IgA $)^{6-8}$.

The $I g H$ constant region contains multiple constant $\left(C_{H}\right)$ genes whose transcription initiates at isotype-specific promoters, called I promoters ${ }^{6}$. The I promoters are largely controlled by the super-enhancer $3^{\prime} \mathrm{RR}\left(3^{\prime}\right.$ Regulatory Region), composed of four enhancers (hs3a, hs1-2, hs3b and hs4), located downstream of the IgH locus ${ }^{9}$.

Transcription from I promoters is induced upon antigen encounter and signaling from other immune cell types $^{6}$. I-derived transcription elongates across highly repetitive sequences, called switch $(S)$ sequences, and generates secondary structures facilitating recruitment of the enzyme AID $\left(e . g{ }^{10-12}\right)$. AID initiates DNA cleavage at the universal donor $S \mu$ region and the activated downstream $S$ region. Ligation of the two $S$ regions brings the downstream constant region into proximity of the rearranged VDJ gene segment, ultimately leading to the expression of a new isotype $e^{6,7}$.

Seminal studies showed that B cells activated by a given signal are transcriptionally committed towards the activated isotype(s) prior to recombination to that particular isotype $(\mathrm{s})^{13-15}$. This pre-switch "transcriptional commitment" model has since been confirmed by various mutational studies targeting I promoters $\left(e . g .{ }^{16-19}\right)$. However, it is unknown whether initially non-activated I promoters are irreversibly silent or if they can be activated when committed B cells are subsequently exposed to inducers promoting their activation.

By using the IgA-committed B cell line $\mathrm{CH} 12$, we show that non-committed I promoters are not irreversibly silent. Following deletion of the committed I $\alpha$ promoter, activation of the I promoter responding to the initial activating cytokine is favored. 
A

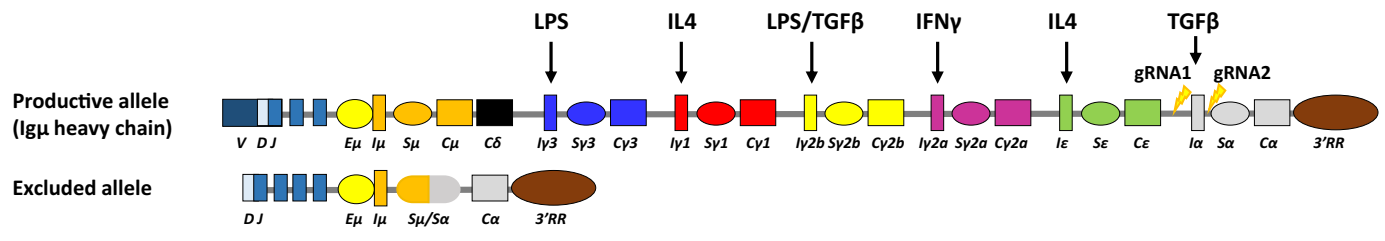

B

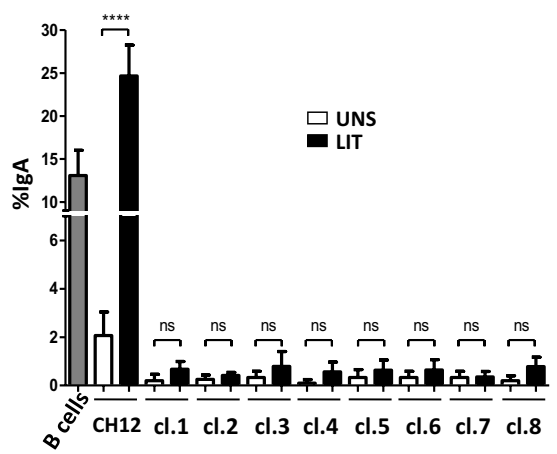

C

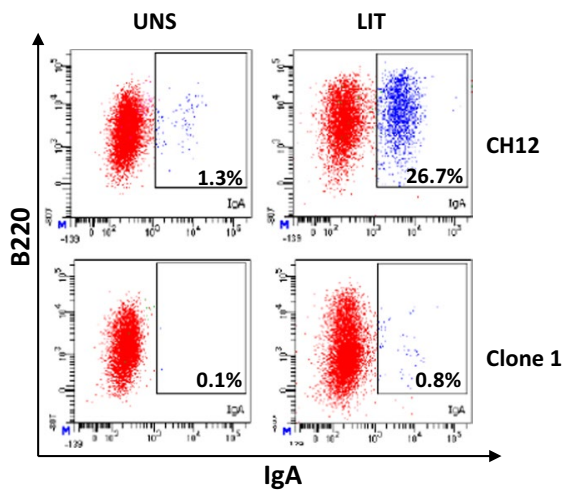

Figure 1. Deletion of I $\alpha$ promoter/exon inhibits CSR to IgA. (A) Schematic structure of the IgH locus in $\mathrm{CH} 12 \mathrm{~F} 3-2$ line. The non-expressed allele is a partially rearranged $\mathrm{DJ}_{\mathrm{H}}$ allele that underwent $\mathrm{S} \mu / \mathrm{S} \alpha$ recombination, thus deleting all upstream inducible I promoters. The mitogen and cytokines inducing the different I promoters are indicated on top. The sites flanking I $\alpha$ promoter/exon targeted by the gRNAs are indicated with arrows. The $\mathrm{E} \mu / \mathrm{I} \mu$ enhancer/promoter between the variable and the constant regions, and the $3^{\prime} \mathrm{RR}$ super-enhancer downstream of the locus are shown. (B) Flow cytometry analysis of I $\alpha$-deleted clones. The 8 clones obtained by CRIPR/Cas9 were analyzed by FACS for IgA surface expression. The parental CH12 line was used as a control prior to (UNS) and following LIT (LPS + IL4 + TGF $\beta$ ) stimulation. LIT-activated splenic B cells were also included as a control $(n=3)$. (C) Representative FACS plot obtained with CH12 cells and an I $\alpha$-deleted clone $\left(n^{\circ} 1\right)$, before and following LIT stimulation.

\section{Results}

CRISPR/Cas9-mediated deletion of the l $\alpha$ promoter in the $\mathrm{CH} 12 \mathrm{F3}-2$ cell line. The murine cell line $\mathrm{CH} 12$ is derived from the CH12.LX lymphoma cell line. This cell line is transcriptionally committed to the I $\alpha$ promoter, which has basal activity even in the absence of stimulation, and activated $\mathrm{CH} 12$ cells switch exclusively to Ig $\mathrm{A}^{20,21}$. Throughout this study, we used the sub-clone CH12F3-2 ${ }^{22}$ (hereafter called CH12 line or cells).

To investigate the effect of deleting the committed I $\alpha$ promoter on activation of upstream I promoters and CSR, we designed two CRISPR/Cas9 guide RNAs specifically targeting the I $\alpha$ promoter/exon (Fig. 1A). Because in $\mathrm{CH} 12$, the non-productive allele has already undergone $\mathrm{S} \mu / \mathrm{S} \alpha$ recombination ${ }^{22,23}$ (Supplementary Fig. S1A), which deleted all I promoters except for the E $\mu / \mathrm{I} \mu$ enhancer/promoter (Fig. 1A), the gRNAs target exclusively the productive allele. PCR screening and sequencing identified eight clones with the desired deletion (Supplementary Fig. S1A-D).

FACS analyses showed that in response to LIT (LPS + IL4 + TGF $\beta$ ), CH12 cells undergo robust CSR to IgA to levels higher than in activated splenic B cells (Fig. 1B). As expected, none of the I $\alpha$-deleted clones switches to IgA (Fig. 1B,C). We checked on three random mutant clones that no trans-splicing occurred between the VDJ exon of the productive allele and the C $\alpha$ region of the non-productive allele (Supplementary Fig. S1E).

Switch transcription and CSR in activated $\mathrm{CH} 12$ cells and deletion clones. To determine if CSR occurs in the absence of the committed I $\alpha$ promoter, we first tested switching under stimulation conditions known to induce switching in primary B cells; LPS stimulation induces CSR to IgG3 and IgG2b, LPS + IL4 to IgG1 and IgE, LPS + IFN $\gamma$ to IgG2a, and LIT to IgG2b and IgA.

FACS analysis revealed that, in contrast to activated splenic B cells, in CH12 as well as in all I $\alpha$-deleted clones, LPS, LPS + IFN $\gamma$ and LPS + IL4 failed to induce CSR to IgG3, IgG2a and IgG1, respectively. None of these stimuli induced CSR to IgA, as expected (Fig. 2A-C, Supplementary Figs. S2 and S3). These results were confirmed in three random clones by RT-qPCR quantification of post-switch transcripts ${ }^{24}$ (Supplementary Fig. S4).

Quantification of pre-switch transcripts in unstimulated (UNS) cells revealed that transcripts levels were higher in deletion clones than in $\mathrm{CH} 12$, except for S $\gamma 2 \mathrm{a}$ (Fig. 2D-F, Supplementary Fig. S5). Moreover, with the exception of $S \varepsilon$ transcripts, whose levels increased in activated I $\alpha$-deleted clones (Fig. 2F), switch transcripts were not further induced following stimulation (Fig. 2D-F).

We conclude that deletion of the committed I $\alpha$ promoter up-regulates most non-committed I promoters. 
A

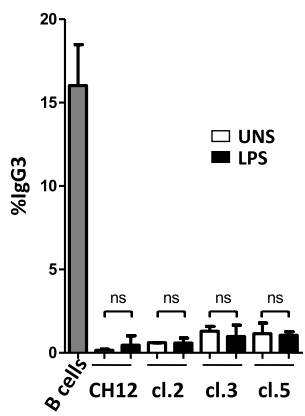

D

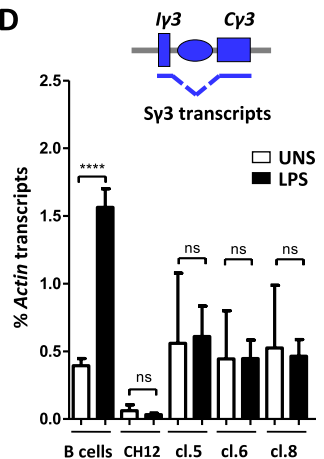

B

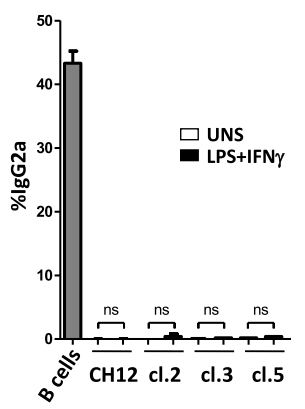

C

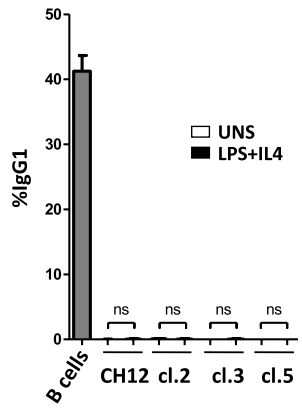

E

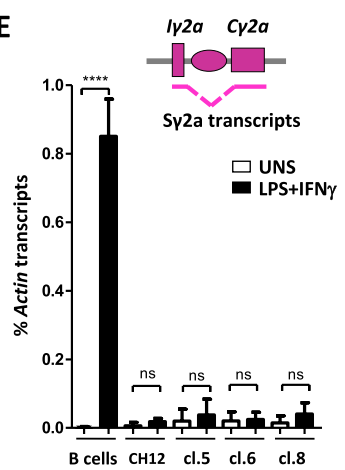

$\mathbf{F}$

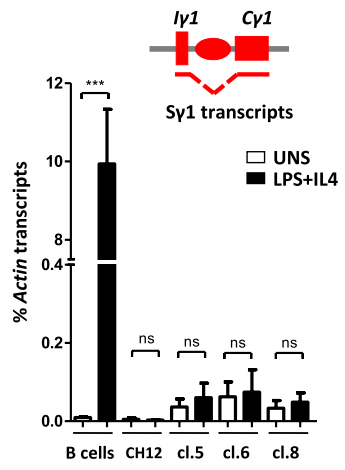

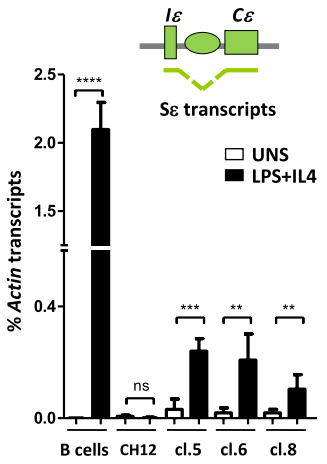

Figure 2. I $\alpha$-deleted clones fail to undergo CSR following specific stimulation. (A-C) CH12 cells, three I $\alpha$ deleted clones, and splenic B cells were activated by LPS (A), LPS + IFN $\gamma(\mathbf{B})$ or LPS + IL4 (C) and stained for IgG3, IgG2a and IgG1, respectively. Representative plots are shown for unstimulated (UNS) and activated CH12 cells, I $\alpha$-deleted clones (clone 5) and primary B cells. (D-F) RT-qPCR quantification of pre-switch transcripts (Sx transcripts) in unstimulated and day 2 activated splenic B cells, CH12 cells or clones 5, 6 and 8 in response to LPS (S $\gamma 3)(\mathbf{D})$, to LPS + IFN $\gamma(\mathrm{S} \gamma 2 \mathrm{a})(\mathbf{E})$, or to LPS + IL4 $(\mathrm{S} \gamma 1$ and $\mathrm{S} \varepsilon)(\mathbf{F})(\mathrm{n}=3)$.

The $\mathrm{I} \gamma 2 \mathrm{~b}$ promoter is induced in response to LIT but not LPS stimulation in $\mathrm{CH} 12$. In primary $\mathrm{B}$ cells, CSR to IgG2b is induced in response to LPS or LIT. We asked if CSR to IgG2b in CH12 cells is induced by either stimulus or only in response to the inducer of the committed isotype (i.e. LIT).

FACS analysis revealed that, unlike primary B cells, LPS stimulation did not induce CSR to IgG2b in either $\mathrm{CH} 12$ or I $\alpha$-deleted clones (Fig. 3A). While in response to LIT, CH12 cells also failed to switch to IgG2b, $\mathrm{I} \alpha$-deleted cells underwent significant switching to IgG2b (Fig. 3B). Although they varied from clone to clone, the levels of CSR to IgG2b in mutant clones were always lower compared to CSR to IgA in CH12 cells (Figs. 1B,C and $3 \mathrm{~B}$ ). Accordingly, $\mathrm{I} \mu-\mathrm{C} \gamma 2 \mathrm{~b}$ transcripts levels increased following LIT stimulation only (Supplementary Fig. S6). Moreover, higher levels of $S \gamma 2 \mathrm{~b}$ pre-switch transcripts were detected in LIT-stimulated clones compared to LPS-stimulated counterparts (Fig. 3C-E). Surprisingly, unlike primary B cells where LPS induces $\mathrm{S} \gamma 2 \mathrm{~b}$ transcription, LPS repressed S $\gamma 2 \mathrm{~b}$ transcription in $\mathrm{CH} 12$ line as well as in all Io-deleted clones (Fig. 3C).

This data shows that $\mathrm{S} \gamma 2 \mathrm{~b}$ transcription and subsequent CSR to IgG2b are induced in I $\alpha$-deleted clones, but only in response to the inducer of the committed isotype (LIT).

Differential induction of switch transcription and CSR in the presence of TGF- $\beta$. The unexpected finding that IgG2b only responds to LIT stimulation suggested to us that the I $\gamma 2 \mathrm{~b}$ promoter responds differently in the CH12 line versus primary B cells. Given that high switching levels to IgA can be achieved when activating CH12 cells with anti-CD40 + IL4 + TGF $\beta$ (CIT) $)^{21,22}$, we wondered if and how this stimulus would impact CSR to IgG2b.

In CH12, cells switched at higher levels to IgA under CIT than LIT (Figs. 1B and 4) and a low percentage of cells switched to IgG2b in the presence of CIT (Figs. 3B and 4). Similarly, In I $\alpha$-deleted clones, switching to IgG2b was considerably higher with CIT than with LIT (Fig. 4). Given the effect of CIT on CSR to IgG2b, we analyzed CSR to other isotypes in CIT-treated cells. We found that switching to IgG3 occurred at varying efficiencies but that switching levels were higher in response to CIT than to LIT (Fig. 4). Switching to IgG1 occurred only with CIT while CSR to IgG2a was undetectable regardless of the stimulation (Fig. 4).

We then asked if the increment in switching with CIT, as compared to LIT, was accompanied by an increase in switch transcription. While $\mathrm{S} \gamma 2 \mathrm{~b}$ pre-switch transcripts levels increased upon CIT stimulation in $\mathrm{CH} 12$ cells, there were no differences in transcript levels between LIT and CIT in deletion clones, whereas in splenic B cells, S $\gamma 2 \mathrm{~b}$ transcripts levels were higher with LIT (Fig. 5A). Surprisingly, while FACS detected higher CSR to IgA with CIT, S $\alpha$ transcripts levels in CH12 cells were higher with LIT than with CIT, but there was no difference in activated splenic B cells (Fig. 5B). I $\alpha$-deleted clones, as expected, did not produce S $\alpha$ transcripts. 

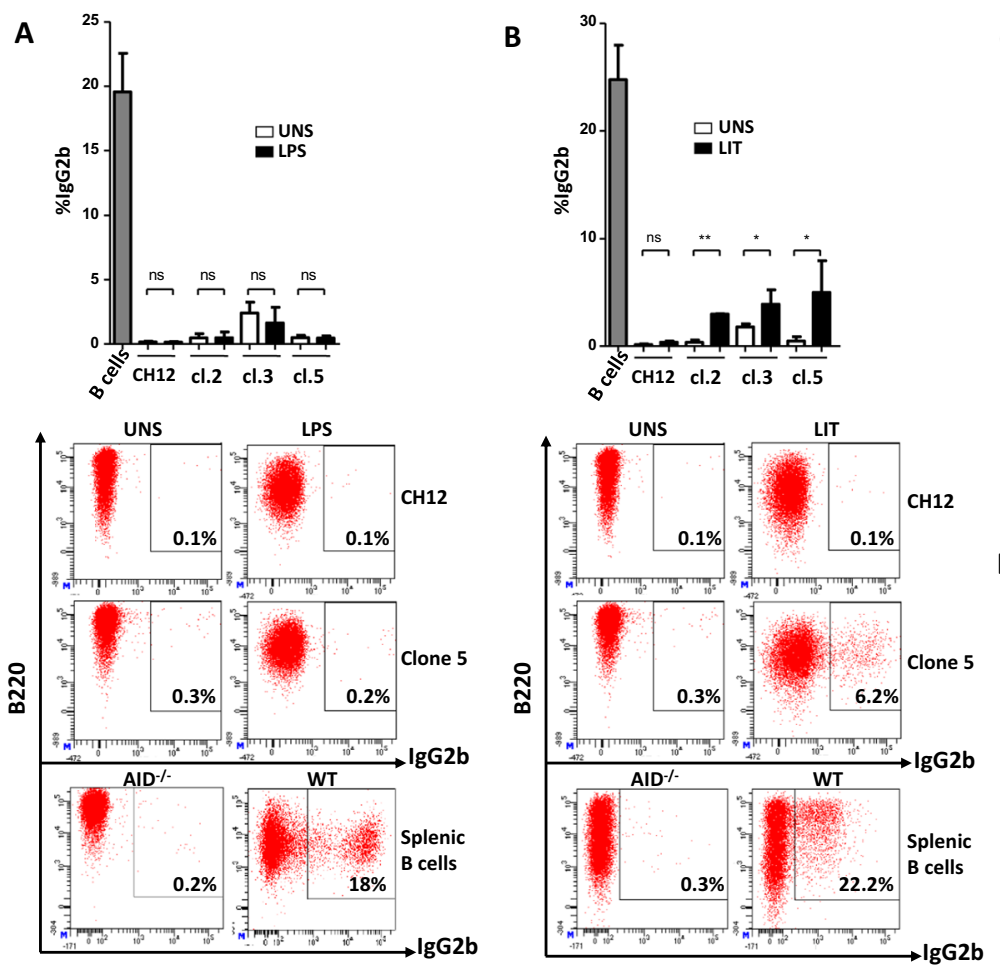

C

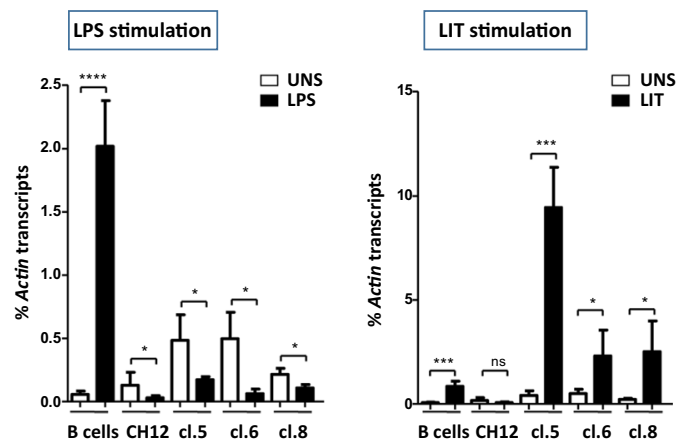

Figure 3. CSR to IgG2b is partially restored in response to TGF- $\beta$ but not to LPS stimulation. (A,B) CH12 cells, three I $\alpha$-deleted clones (2, 3, and 5) and splenic B cells were activated by LPS (A) or LIT (B) for 4 days, and stained for IgG2b. Representative plots are shown for unstimulated (UNS), activated CH12 cells, I $\alpha$-deleted clones (clone 5) and primary B cells. (C,D) RT-qPCR quantification of S $\gamma 2 \mathrm{~b}$ pre-switch transcripts levels in unstimulated clones 5, 6 and 8 and in response to LPS (C) or to LIT (D) (day 2). (E) Comparison of S $\gamma 2 \mathrm{~b}$ preswitch transcripts levels in I $\alpha$-deleted clones 5, 6 and 8, following LPS and LIT stimulation $(\mathrm{n}=3)$.
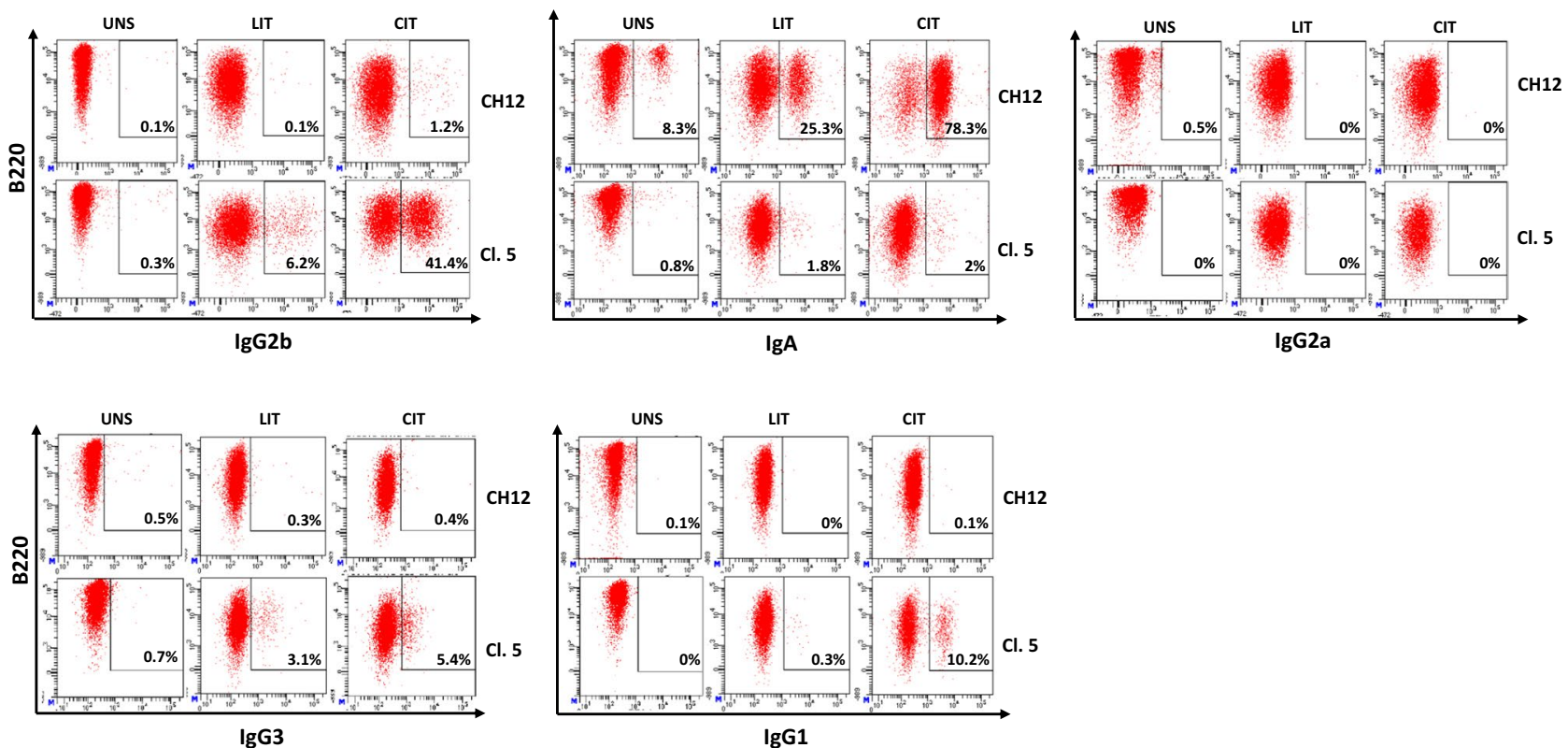

Figure 4. Differential induction of CSR in response to LIT and CIT. CH12 cells and three I $\alpha$-deleted clones (3, 5 and 8) were activated by LIT or CIT for 4 days, and stained for the indicated isotypes. Representative plots are shown for activated $\mathrm{CH} 12$ cells and I $\alpha$-deleted clones (clone 5$)(\mathrm{n} \geq 3)$.

Although there was increased CSR to IgG3 with CIT and no switching to IgG2a, regardless of stimulation, $S \gamma 2$ a (with the exception of clone 5) and $S \gamma 3$ transcripts levels were comparable between LIT- and CIT-activated deletion clones (Fig. 5C,D). 
A

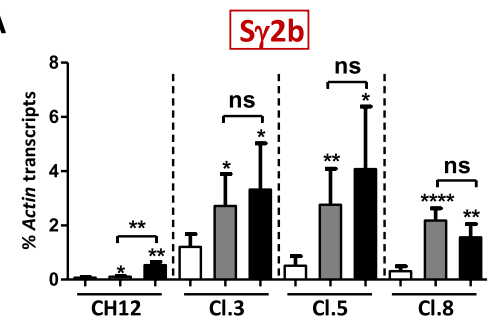

c

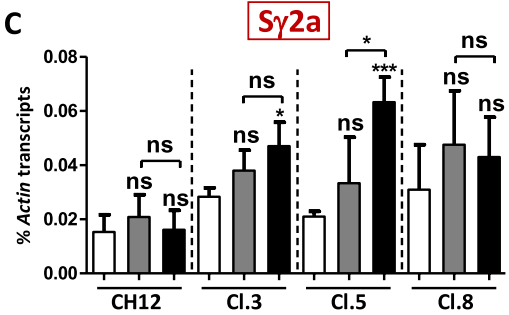

$$
\text { E }
$$

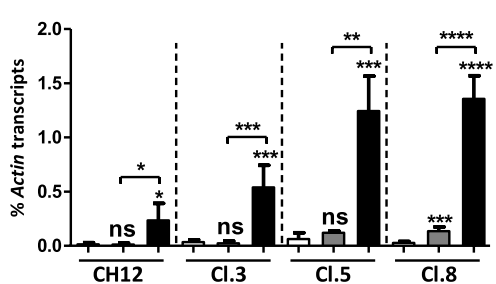

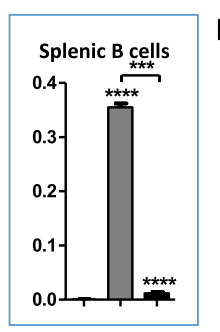

B

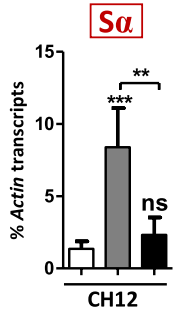

D
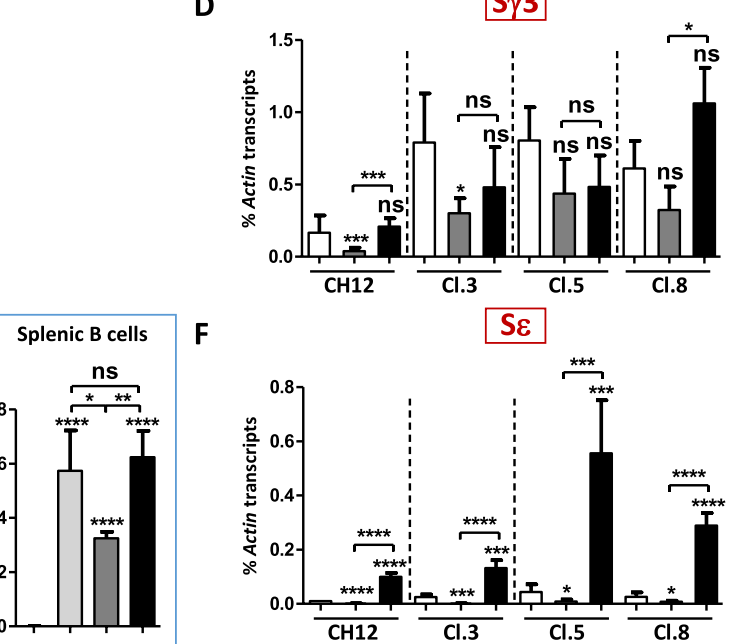

F

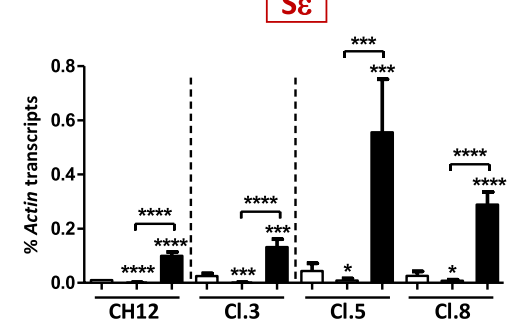

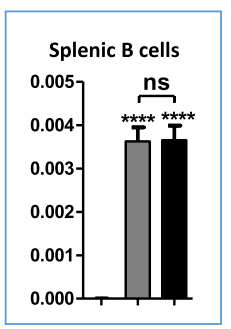

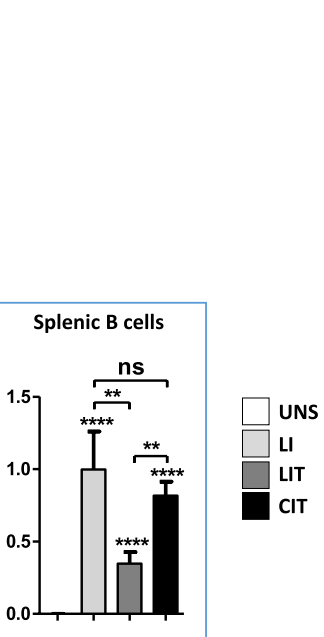

Figure 5. Differential induction of switch transcription in response to LIT and CIT. (A-F) RT-qPCR quantification of $S \gamma 2 b(\mathbf{A}), S \alpha(\mathbf{B}), S \gamma 2 \mathrm{a}(\mathbf{C}), S \gamma 3(\mathbf{D}), S \gamma 1$ (E), and $S \varepsilon(\mathbf{F})$ pre-switch transcripts levels in $\mathrm{CH} 12$ cells and I $\alpha$-deleted clones 3, 5 and 8 following LIT or CIT stimulation. Transcripts levels in splenic B cells activated with LIT and CIT for S $\gamma 2 \mathrm{~b}(\mathbf{A})$ and $S \alpha(\mathbf{B})$, and with LPS + IL4 (LI), LIT and CIT for S $\gamma 1$ and S $\varepsilon(\mathbf{E}, \mathbf{F})$ are boxed $(n \geq 3)$.

In $\mathrm{CH} 12$ cells and in deletion clones, LIT repressed $S \varepsilon$, but not $S \gamma 1$ transcription, except for clone 8 in which $\mathrm{S} \gamma 1$ transcripts increased. CIT induced $\mathrm{S} \gamma 1$ and $S \varepsilon$ transcription (Fig. 5E,F and Supplementary Fig. S7). In splenic B cells, CIT was just as efficient as LPS + IL4 (LI) in inducing S $\gamma 1$ and S $\varepsilon$ transcription, and the levels were higher than with LIT (Fig. 5E,F).

Thus, in the absence of the committed I $\alpha$ promoter, CSR to IgG2a does not take place, and while switching to IgG1 occurs with CIT only, switching to IgG2b and IgG3 occurs in response to both CIT and LIT. However, switching was constantly higher in response to CIT. Surprisingly, switching efficiency did not always correlate with switch transcripts levels.

Lack of correlation between Aicda transcripts levels and CSR efficiency. Since the enzyme AID is absolutely required to initiate CSR, we wondered if the lack of correlation between switch transcription and CSR in some cases is due to lower expression of the Aicda gene, encoding AID.

We found that Aicda transcripts levels were higher in $\mathrm{CH} 12$ cells and derived clones compared to primary B cells, both in unstimulated and stimulated conditions (Fig. 6). LI (LPS + IL4) did not induce Aicda transcription in $\mathrm{CH} 12$ and I $\alpha$-deleted clones, but only in primary B cells (Fig. 6). In contrast to LI, LIT and CIT efficiently induced Aicda gene in all cells (Fig. 6).

Therefore, while absence of switching to $\mathrm{S} \varepsilon$ in $\mathrm{CH} 12$ and deletion clones following LI stimulation correlates with the lack of induction of Aicda gene, the same is not true for the differences in CSR to IgG2b and IgA in the presence of LIT and CIT.

Increased transcription of hs1-2 enhancer in response to LIT but not CIT. Enhancer RNAs (eRNAs) are produced at the $3^{\prime} \mathrm{RR}$ following activation of splenic B cells and are a hallmark of $3^{\prime} \mathrm{RR}$ activity $\mathrm{y}^{25-27}$. We then wondered if the non-correlation between switch transcription and CSR, in the cases where there is similar activation of Aicda, could be explained by differences in the transcriptional activity of the $3^{\prime} \mathrm{RR}$. In order to test this, we quantified hs3a, hs1-2 and hs3b transcripts levels.

eRNAs levels were comparable between unstimulated $\mathrm{CH} 12$ cells and deletion clones, and were higher than in splenic B cells (Fig. 7). For both $\mathrm{CH} 12$ cells and deletion clones, while hs3a and hs3b eRNAs levels did not significantly vary with LIT and CIT (despite some clonal variation) (Fig. 7), there was a consistent trend towards increased hs1-2 transcription with LIT (Fig. 7). 
$\mathrm{CH} 12$

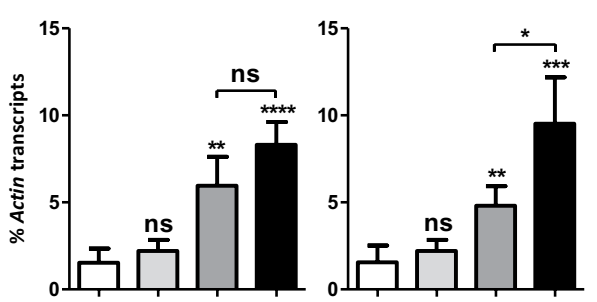

Clone 5

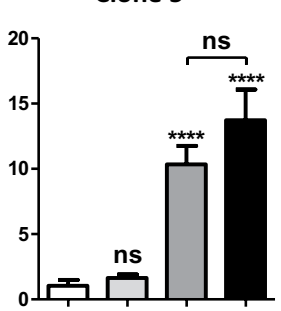

Clone 8

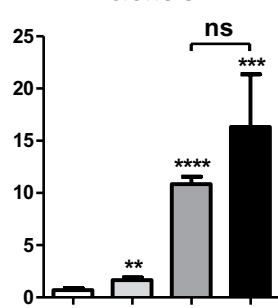

Splenic B cells

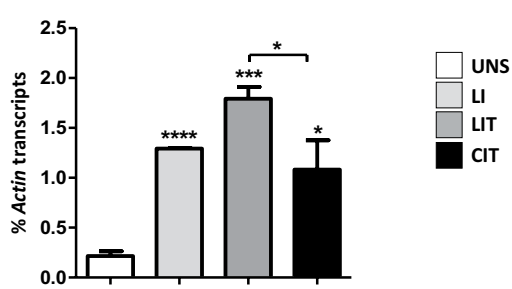

Figure 6. Stimulus-dependent induction of Aicda gene transcription. CH12 cells, three I $\alpha$-deleted clones (3, 5 and 8) and splenic B cells were activated with LPS + IL4 (LI), LIT or CIT for 2 days. Total RNAs were collected from unstimulated and activated cells and Aicda transcripts were quantified by RT-qPCR. Actin transcripts were used for normalization $(n \geq 3)$.

hs3a

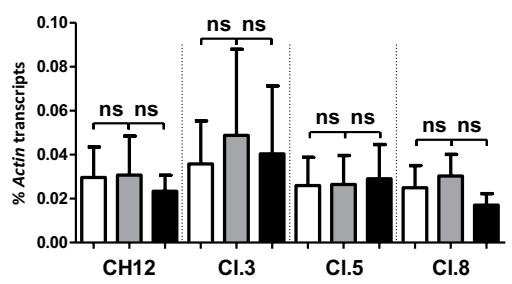

hs1-2

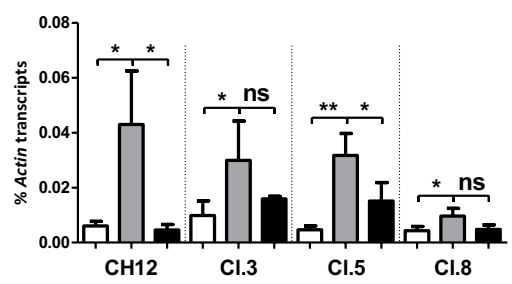

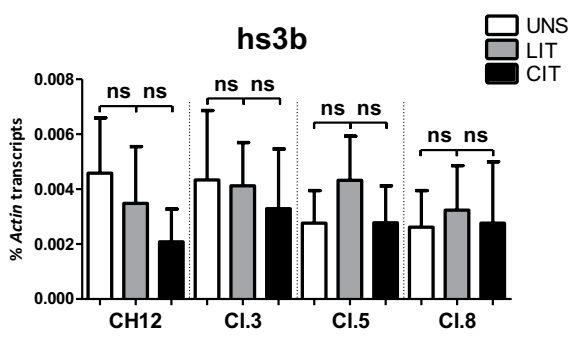

Figure 7. $3^{\prime} \mathrm{RR}$ transcripts levels upon LIT and CIT stimulations. RT-qPCR quantification of eRNAs levels of hs3a, hs1-2 and hs3b enhancers. Total RNAs were extracted from unstimulated CH12 cells and I $\alpha$-deleted clones $(3,5$, and 8$)$ at day 2 post-stimulation. Actin transcripts were used for normalization, and (-RT) controls were included throughout $(\mathrm{n} \geq 3)$.

While enhanced transcription of hs1-2 in response to LIT correlates with low levels of $S \gamma 1$ and S $\varepsilon$ transcripts, high levels of $S \gamma 2 b$ and $S \alpha$ transcription were detected in the presence of LIT. Therefore, increased transcription of hs1-2 enhancer cannot alone explain the dissociation between switch transcription and CSR.

\section{Discussion}

The CH12 cell line is widely used to study various aspects of CSR $\left(\right.$ e.g. $\left.{ }^{11,12,22,23,28}\right)$. However, transcriptional activity of its I promoters had not yet been studied. We investigated the transcriptional status of the non-committed I promoters and CSR levels in the presence or absence of the initially committed I $\alpha$ promoter, following various stimulations classically used for primary B cells and/or $\mathrm{CH} 12$.

Treatment of the parental CH12 line with LPS, LI or LPS + IFN $\gamma$ did not activate any of the I promoters. However, the block was not irreversible because, upon deletion of the committed I $\alpha$ promoter, switch transcripts levels of all isotypes (with the possible exception of $S \gamma 2 a$ ) increased in unstimulated cells. This indicates that simple deletion of the committed promoter enabled other I promoters to acquire a relatively open chromatin state.

Upon stimulation of I $\alpha$-deleted clones, the I promoters displayed different responses. For instance, while I $\gamma 1$ was induced by CIT only, I $\varepsilon$ was induced by LI and, more strongly, by CIT, but was repressed by LIT. Because both LIT and CIT contain TGF- $\beta$, strong induction of I $\varepsilon$ and of I $\gamma 1$ only in the presence of CIT was surprising. It has been shown that, in splenic B cells, TGF $\beta$ induces the Id 2 repressor, which antagonizes binding of basic helix-loop-helix E2A and PAX5 transcription factors, precluding activation of the I $\varepsilon$ promoter (E2A in the case of $\mathrm{I} \gamma 1)^{29}$. We found that $S \gamma 1$ and $S \varepsilon$ transcripts levels were reduced in splenic B cells activated with LIT, compared to CIT. Together, these findings indicate that TGF $\beta$ impacts $S \gamma 1$ and $S \varepsilon$ transcription in the presence of LPS, but not of anti-CD40. This suggests that signaling through CD40 somehow circumvents TGF $\beta$-induced Id2-mediated suppression of transcription factors activity. The Toll-like receptor 4 (TLR4, which binds LPS) and CD40 preferentially trigger the classical and the alternative NF- $\kappa B$ signaling pathways, respectively ${ }^{30,31}$. Nonetheless, LPS can activate both pathways through binding to both TLR4 and surface IgM ${ }^{32,33}$. Given that all I promoters (except I $\mu$ ) and the $3^{\prime} \mathrm{RR}$ have NF- $\mathrm{BB}$ binding sites ${ }^{6,9,34}$, this suggests that the cross-talks between TGF $\beta$ and LPS pathways on one hand, and TGF $\beta$ and CD40 ligand pathways on the other hand, have more complex transcriptional outcomes than previously thought.

CIT induced higher levels of CSR than LIT, but there was not always a correlation between increased CSR and switch transcription as illustrated by increased S $\alpha$ transcription in CH12 cells in the presence of LIT, and similar $\mathrm{S} \gamma 2 \mathrm{~b}$ transcript levels in deletion clones under the two stimulations. This suggested that other mechanisms might be involved. We investigated transcription of Aicda gene under different stimulations. We showed that LIT and CIT, but not LI, efficiently induced Aicda in CH12 cells and deletion clones. Nonetheless, there was not always a correlation between switching efficiency and Aicda induction. On one hand, the failure to switch to $S \varepsilon$ in response to LI could be due to non-induction of Aicda, but also to low levels of S $\varepsilon$ transcripts, or to a conjunction of both. 


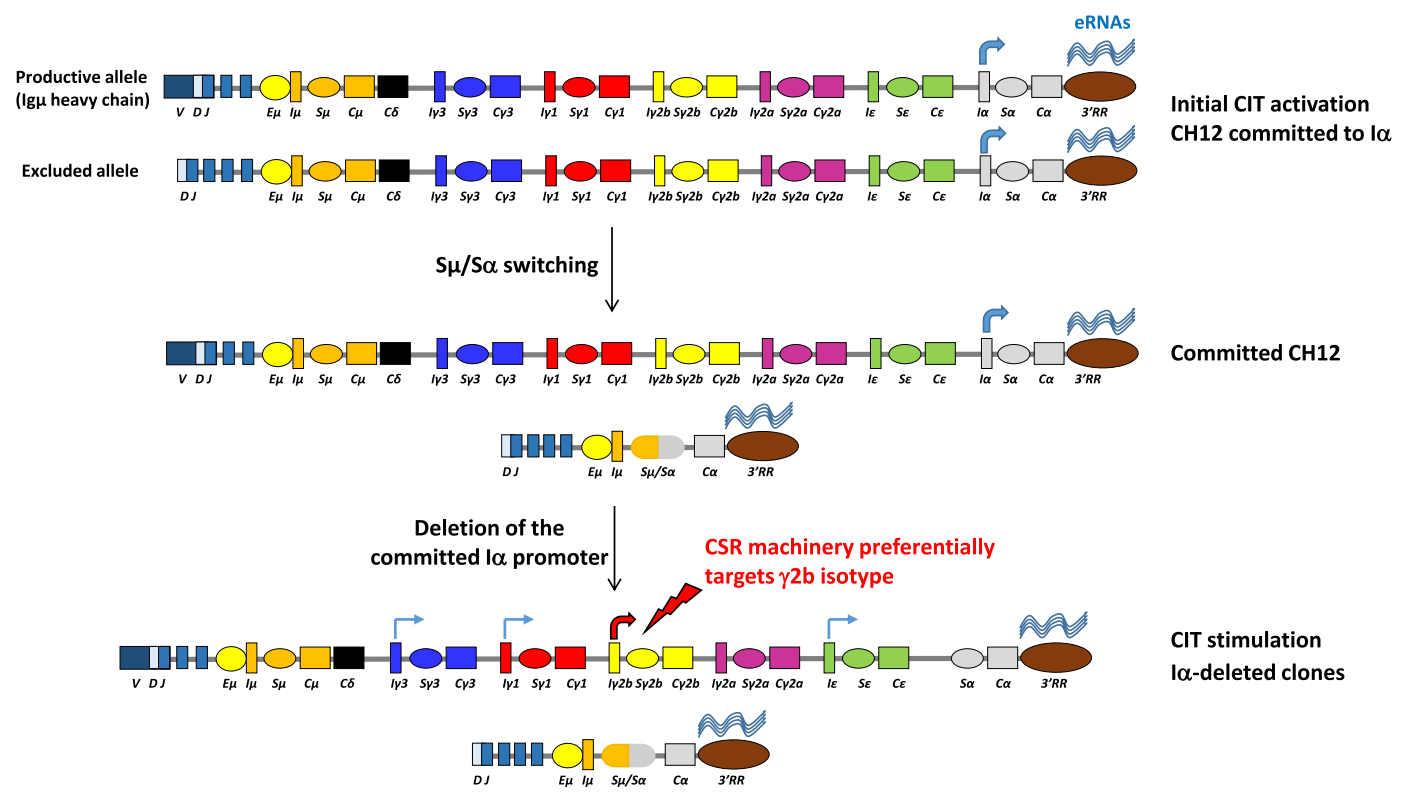

Figure 8. Model of the natural history of the $\mathrm{CH} 12$ line. Initial activation of the original B cell clone likely took place in the context of a T-dependent response involving TGF- $\beta$ and CD40. This led to commitment to I $\alpha$ promoter and induction of $3^{\prime} \mathrm{RR}$ transcription on both alleles, and subsequent switching on the non-expressed allele. The CSR machinery retained somehow memory of the initial activating signal (CIT). The I $\alpha$ promoter and the $3^{\prime} \mathrm{RR}$ remain active in the committed $\mathrm{CH} 12$ line. Upon deletion of the committed I $\alpha$ promoter, I $\gamma 2 \mathrm{~b}$ normally induced with either LPS or TGF- $\beta$, is only induced in response to TGF- $\beta$, but the highest switching levels to IgG2b are preferentially achieved with the initial signal (CIT).

On the other hand, CIT induced higher levels of CSR to IgA in CH12 cells, and to IgG2b in deletion clones, than LIT but this did not correlate with a substantial increase in Aicda gene induction. Therefore, AID levels per se cannot explain the large difference in switching efficiency in response to CIT versus LIT. We suggest that factors acting downstream of AID may be more strongly induced by CIT than LIT.

Transcriptional activity of the $3^{\prime} \mathrm{RR}$ could also account for the complex transcriptional pattern of I promoters. Given that unstimulated $\mathrm{CH} 12$ cells and deletion clones expressed similar eRNAs levels, deletion of the committed promoter did not affect 3'RR activity. Additionally, eRNAs levels in $\mathrm{CH} 12$ were higher compared to primary B cells, and were insensitive to CIT. This suggests that eRNAs have reached a level sufficient for activation of specific I promoters. In this regard, it should be noted that the $\mathrm{CH} 12$ line has already switched on the non-expressed allele, i.e., it has experienced activation that induced the $3^{\prime} \mathrm{RR}$ on both alleles (Fig. 8).

Remarkably, hs1-2 transcription could be further induced by LIT, and this correlated with low levels of S $\gamma 1$ and $S \varepsilon$ transcripts. This pattern is consistent with a model in which $\mathrm{I} \gamma 1$ and $\mathrm{I} \varepsilon$ promoters compete with highly transcribed $3^{\prime} \mathrm{RR}$ enhancers for transcription factors ${ }^{27}$. However, the activation patterns of I $\gamma 2 \mathrm{~b}$ (similarly induced by LIT and CIT) and I $\alpha$ (induced by LIT but not by CIT) suggest different or additional mechanisms. Various non-mutually exclusive mechanisms could be involved including local factors specific to I promoters (combinatorial effect of transcription factors, cis-acting elements...), preferential interactions of individual $3^{\prime} \mathrm{RR}$ enhancers with I promoters ${ }^{35,36}$, and physical proximity to the $3^{\prime} \mathrm{RR}$. The finding that heightened transcription of hs1-2 enhancer with LIT correlates with reduced CSR to IgG2b and IgA may indicate that hs1-2 enhancer competes with $S \gamma 2 b$ and $S \alpha$ for AID. Recruitment of AID by super-enhancers has been demonstrated ${ }^{37,38}$ and there is some evidence that AID could target the $3^{\prime} R^{25}$.

A remarkable finding of this study concerns the switch levels to IgG2b in deletion clones that are considerably higher in response to CIT than to LIT. This was unexpected, and as mentioned, cannot be due to higher switch transcripts levels or to $3^{\prime} \mathrm{RR}$ eRNAs or AID levels, but may suggest that activation of the initial B cell clone (that gave rise to $\mathrm{CH} 12$ line) took place in the context of a T-dependent response, which activated the CD40 pathway. Moreover, the $\mathrm{CH} 12$ line has already undergone switching on the excluded allele and may therefore represent an advanced state of commitment. Importantly, in the absence of the committed promoter, particularly in the presence of CIT, $\mathrm{S} \gamma 2 \mathrm{~b}$ transcription is more strongly induced than in primary B cells, and, of all isotypes, the highest levels of switching occur towards IgG2b specifically. Thus, in terms of both transcription and switching, the $\gamma 2 \mathrm{~b}$ isotype appears to be the preferential target of CIT, i.e. the likely initial committing signal. We have recently shown that in the majority of TGF $\beta$-activated splenic B cells, the I $\gamma 2 \mathrm{~b}$ and I $\alpha$ promoters compete for the $3^{\prime} \mathrm{RR}^{39}$. Although we cannot ascertain if commitment to the I $\alpha$ promoter in the original CH12 line took place following co-activation of $\mathrm{I} \gamma 2 \mathrm{~b}$ and I $\alpha$ or single activation of I $\alpha$, the CSR pattern in deletion clones raises the possibility that commitment to the I $\alpha$ promoter has rewired the CSR machinery so that, even after deletion of I $\alpha$, it targets I $\gamma 2 \mathrm{~b}$ and there is optimal switching in response to the initial signal (Fig. 8). Whether this coincides with CIT-induced formation of specialized nuclear compartments such as transcription factories ${ }^{40}$ that would facilitate I $\gamma 2 \mathrm{~b}-3^{\prime} \mathrm{RR}$ interactions and recruitment of AID remains to be explored. 
In conclusion, we showed that initial commitment to the I $\alpha$ promoter in $\mathrm{CH} 12$ cells blocks transcriptional activation of other promoters. However, the block is not irreversible. Commitment to a particular isotype appears to target the CSR machinery towards a pathway in which the stimulating cytokine plays a key role so that in the absence of the committed promoter, activation of the I promoter responding to the initial signal is favored. It would be interesting to explore if the same is true for primary B cells.

\section{Material and Methods}

Cell culture. CH12 and primary splenic B cells (from 129Sv1 mouse strain) were cultured in RPMI media supplemented with $10 \%$ heat inactivated serum, $10 \mathrm{mM}$ HEPES, $1 \mathrm{mM}$ sodium pyruvate, $100 \mathrm{U} / \mathrm{mL}$ Penicillin, $100 \mathrm{U} / \mathrm{mL}$ Streptomycin, $50 \mu \mathrm{M} \beta$-mercaptoethanol, $1 \mathrm{x}$ non-essential amino acids.

$\mathrm{CH} 12$ cells were stimulated for 2 or 4 days at a density of $10^{5}$ cells $/ \mathrm{ml}$, in the presence of $50 \mu \mathrm{g} / \mathrm{ml}$ of LPS (Sigma) (LPS stimulation); $50 \mu \mathrm{g} / \mathrm{ml} \mathrm{LPS} \mathrm{and} 20 \mathrm{ng} / \mathrm{ml}$ IFN- $\gamma$ (R\&D) (IFN- $\gamma$ stimulation); $50 \mu \mathrm{g} / \mathrm{ml} \mathrm{LPS}$ and $25 \mathrm{ng} / \mathrm{ml} \mathrm{IL4} \mathrm{(eBiosciences)} \mathrm{(IL4} \mathrm{stimulation);} 50 \mu \mathrm{g} / \mathrm{ml} \mathrm{LPS,} 10 \mathrm{ng} / \mathrm{ml} \mathrm{IL4}$, and $2 \mathrm{ng} / \mathrm{ml} \mathrm{TGF \beta} \mathrm{(R \& D)} \mathrm{(LIT} \mathrm{stim-}$ ulation); $1 \mu \mathrm{g} / \mathrm{ml}$ anti-CD40 (eBiosciences), $10 \mathrm{ng} / \mathrm{ml} \mathrm{IL4}$, and $2 \mathrm{ng} / \mathrm{ml}$ TGF $\beta$ (CIT stimulation). Purification and stimulation of primary splenic B cells were as described (ref. ${ }^{40}$ ).

Molecular cloning. The gRNAs oligonucleotides were phosphorylated with T4 Polynucleotide Kinase (Thermo Scientific) and annealed. Afterwards, they were ligated with BsaI or BbsI digested pX333 plasmid (Addgene). Confirmed cloning products were used as template to PCR amplify the gRNA cassette with primers gRNA-M $l u I F w$ and gRNA-M $l u I R e v$, and the PCR fragment was cloned into MluI digested CMV-Cas9-GFP plasmid (Sigma). Correct cloning was diagnosed by restriction digestion and sequencing. All primers are listed in Table S1.

$\mathrm{CH} 12$ cells transfection. $2 \times 10^{6}$ cells were transfected with $2 \mu \mathrm{g}$ of CMV-Cas9-GFP-gRNA by electroporation using program O-006 of the Amaxa Nucleofector II (Lonza) and Amaxa Cell Line Nucleofector kit V. Transfected cells were cultured at $37^{\circ} \mathrm{C}$ for $24 \mathrm{~h}$. GFP-positive cells were single-sorted into 96 -well plates, cultured for seven days and then PCR tested for the presence of the deletion with the appropriate primers and GoTaq Polymerase (Promega), according to the manufacturer's instructions. Deletion clones were checked by sequencing.

RT-qPCR. Total RNAs were collected from non-stimulated or stimulated cells at day 2 or day 4 post-treatment using a commercial kit (Zymo Research). Total RNAs were reverse-transcribed (Invitrogen) and qPCR was performed using Sso Fast Eva Green (BioRad), according to the manufacturer's instructions. Actin transcripts levels were used for normalization and the results are shown as percentage of actin. (-RT) controls were tested for all samples.

Flow cytometry. At day 4 post-stimulation, cells were washed and stained with anti-B220 APC (BioLegend) and either anti-IgG3-FITC (BD-Pharmingen), anti-IgG1-FITC (BioLegend), anti-IgG2b-PE (BioLegend), anti-IgG2a-PE (BioLegend) or anti-IgA-FITC (BD-Pharmingen). Data were obtained on $3 \times 10^{4}$ viable cells by using a Coulter XL apparatus (Beckman Coulter).

Statistical analysis. Results are expressed as mean \pm SD (GraphPad Prism), and overall differences between values from WT and mutant mice were evaluated by a two-tailed $t$ test. The difference between means is significant if $p<0.05(*)$, very significant if $p<0.01(* *)$, and extremely significant if $p<0.001(* * *)$.

\section{Data availability}

Materials, data and associated protocols are promptly available to readers.

Received: 22 July 2019; Accepted: 21 November 2019;

Published online: 06 December 2019

\section{References}

1. Rajewsky, K. Clonal selection and learning in the antibody system. Nature 381, 751-758, https://doi.org/10.1038/381751a0 (1996).

2. Meffre, E., Casellas, R. \& Nussenzweig, M. C. Antibody regulation of B cell development. Nature immunology 1, 379-385, https:// doi.org/10.1038/80816(2000).

3. Schatz, D. G. \& Ji, Y. Recombination centres and the orchestration of V(D)J recombination. Nature reviews. Immunology 11, 251-263, https://doi.org/10.1038/nri2941 (2011).

4. Kumari, G. \& Sen, R. Chromatin Interactions in the Control of Immunoglobulin Heavy Chain Gene Assembly. Advances in immunology 128, 41-92, https://doi.org/10.1016/bs.ai.2015.08.001 (2015).

5. Jung, D., Giallourakis, C., Mostoslavsky, R. \& Alt, F. W. Mechanism and control of V(D)J recombination at the immunoglobulin heavy chain locus. Annual review of immunology 24, 541-570, https://doi.org/10.1146/annurev.immunol.23.021704.115830 (2006).

6. Stavnezer, J., Guikema, J. E. \& Schrader, C. E. Mechanism and regulation of class switch recombination. Annual review of immunology 26, 261-292, https://doi.org/10.1146/annurev.immunol.26.021607.090248 (2008).

7. Chaudhuri, J. \& Alt, F. W. Class-switch recombination: interplay of transcription, DNA deamination and DNA repair. Nature reviews. Immunology 4, 541-552, https://doi.org/10.1038/nri1395 (2004).

8. Yu, K. \& Lieber, M. R. Current insights into the mechanism of mammalian immunoglobulin class switch recombination. Critical reviews in biochemistry and molecular biology 54,333-351, https://doi.org/10.1080/10409238.2019.1659227 (2019).

9. Khamlichi, A. A., Pinaud, E., Decourt, C., Chauveau, C. \& Cogne, M. The $3^{\prime}$ IgH regulatory region: a complex structure in a search for a function. Advances in immunology 75, 317-345 (2000).

10. Yu, K., Chedin, F., Hsieh, C. L., Wilson, T. E. \& Lieber, M. R. R-loops at immunoglobulin class switch regions in the chromosomes of stimulated B cells. Nature immunology 4, 442-451, https://doi.org/10.1038/ni919 (2003).

11. Zheng, S. et al. Non-coding RNA Generated following Lariat Debranching Mediates Targeting of AID to DNA. Cell 161, 762-773, https://doi.org/10.1016/j.cell.2015.03.020 (2015) 
12. Ribeiro de Almeida, C. et al. RNA Helicase DDX1 Converts RNA G-Quadruplex Structures into R-Loops to Promote IgH Class Switch Recombination. Molecular cell 70, 650-662.e658, https://doi.org/10.1016/j.molcel.2018.04.001 (2018).

13. Yancopoulos, G. D. et al. Secondary genomic rearrangement events in pre-B cells: VHDJH replacement by a LINE-1 sequence and directed class switching. The EMBO journal 5, 3259-3266 (1986).

14. Stavnezer-Nordgren, J. \& Sirlin, S. Specificity of immunoglobulin heavy chain switch correlates with activity of germline heavy chain genes prior to switching. The EMBO journal 5, 95-102 (1986).

15. Lutzker, S., Rothman, P., Pollock, R., Coffman, R. \& Alt, F. W. Mitogen- and IL-4-regulated expression of germ-line Ig gamma $2 \mathrm{~b}$ transcripts: evidence for directed heavy chain class switching. Cell 53, 177-184, https://doi.org/10.1016/0092-8674(88)90379-0 (1988).

16. Jung, S., Rajewsky, K. \& Radbruch, A. Shutdown of class switch recombination by deletion of a switch region control element. Science (New York, N.Y.) 259, 984-987, https://doi.org/10.1126/science.8438159 (1993).

17. Zhang, J., Bottaro, A., Li, S., Stewart, V. \& Alt, F. W. A selective defect in IgG2b switching as a result of targeted mutation of the I gamma $2 \mathrm{~b}$ promoter and exon. The EMBO journal 12, 3529-3537 (1993).

18. Harriman, G. R., Bradley, A., Das, S., Rogers-Fani, P. \& Davis, A. C. IgA class switch in I alpha exon-deficient mice. Role of germline transcription in class switch recombination. The Journal of clinical investigation 97, 477-485, https://doi.org/10.1172/jci118438 (1996).

19. Seidl, K. J. et al. Position-dependent inhibition of class-switch recombination by PGK-neor cassettes inserted into the immunoglobulin heavy chain constant region locus. Proceedings of the National Academy of Sciences of the United States of America 96, 3000-3005, https://doi.org/10.1073/pnas.96.6.3000 (1999).

20. Wakatsuki, Y. \& Strober, W. Effect of downregulation of germline transcripts on immunoglobulin A isotype differentiation. The Journal of experimental medicine 178, 129-138, https://doi.org/10.1084/jem.178.1.129 (1993).

21. Nakamura, M. et al. High frequency class switching of an IgM+B lymphoma clone CH12F3 to IgA + cells. International immunology 8, 193-201, https://doi.org/10.1093/intimm/8.2.193 (1996).

22. Ono, S. J. et al. Identification of a stimulus-dependent DNase I hypersensitive site between the Ialpha and Calpha exons during immunoglobulin heavy chain class switch recombination. FEBS letters 467, 268-272, https://doi.org/10.1016/s0014-5793(00)01151-0 (2000).

23. Kim, A., Han, L., Santiago, G. E., Verdun, R. E. \& Yu, K. Class-Switch Recombination in the Absence of the IgH $3^{\prime}$ Regulatory Region. Journal of immunology (Baltimore, Md.: 1950) 197, 2930-2935, https://doi.org/10.4049/jimmunol.1600530 (2016).

24. Li, S. C. et al. Expression of I mu-C gamma hybrid germline transcripts subsequent to immunoglobulin heavy chain class switching. International immunology 6, 491-497, https://doi.org/10.1093/intimm/6.4.491 (1994).

25. Peron, S. et al. AID-driven deletion causes immunoglobulin heavy chain locus suicide recombination in B cells. Science (New York, N.Y.) 336, 931-934, https://doi.org/10.1126/science.1218692 (2012).

26. Braikia, F. Z. et al. Developmental Switch in the Transcriptional Activity of a Long-Range Regulatory Element. Molecular and cellular biology 35, 3370-3380, https://doi.org/10.1128/MCB.00509-15 (2015).

27. Delgado-Benito, V. et al. The Chromatin Reader ZMYND8 Regulates Igh Enhancers to Promote Immunoglobulin Class Switch Recombination. Molecular cell 72, 636-649.e638, https://doi.org/10.1016/j.molcel.2018.08.042 (2018).

28. Zhang, Z. Z. et al. Effect of $\mathrm{CpG}$ dinucleotides within IgH switch region repeats on immunoglobulin class switch recombination. Molecular immunology 66, 284-289, https://doi.org/10.1016/j.molimm.2015.04.005 (2015).

29. Sugai, M. et al. Essential role of Id2 in negative regulation of IgE class switching. Nature immunology 4, 25-30, https://doi. org/10.1038/ni874 (2003)

30. Hayden, M. S. \& Ghosh, S. NF-kappaB, the first quarter-century: remarkable progress and outstanding questions. Genes \& development 26, 203-234, https://doi.org/10.1101/gad.183434.111 (2012).

31. Sun, S. C. The non-canonical NF-kappaB pathway in immunity and inflammation. Nature reviews. Immunology 17, 545-558, https:// doi.org/10.1038/nri.2017.52 (2017)

32. Pone, E. J. et al. BCR-signalling synergizes with TLR-signalling for induction of AID and immunoglobulin class-switching through the non-canonical NF-kappaB pathway. Nature communications 3, 767, https://doi.org/10.1038/ncomms1769 (2012).

33. Xu, Z., Zan, H., Pone, E. J., Mai, T. \& Casali, P. Immunoglobulin class-switch DNA recombination: induction, targeting and beyond. Nature reviews. Immunology 12, 517-531, https://doi.org/10.1038/nri3216 (2012).

34. Pinaud, E. et al. The IgH locus $3^{\prime}$ regulatory region: pulling the strings from behind. Advances in immunology 110, 27-70, https:// doi.org/10.1016/b978-0-12-387663-8.00002-8 (2011).

35. Laurencikiene, J., Deveikaite, V. \& Severinson, E. HS1,2 enhancer regulation of germline epsilon and gamma2b promoters in murine B lymphocytes: evidence for specific promoter-enhancer interactions. Journal of immunology (Baltimore, Md.: 1950) 167, 3257-3265, https://doi.org/10.4049/jimmunol.167.6.3257 (2001).

36. Sellars, M., Reina-San-Martin, B., Kastner, P. \& Chan, S. Ikaros controls isotype selection during immunoglobulin class switch recombination. The Journal of experimental medicine 206, 1073-1087, https://doi.org/10.1084/jem.20082311 (2009).

37. Meng, F. L. et al. Convergent transcription at intragenic super-enhancers targets AID-initiated genomic instability. Cell 159, 1538-1548, https://doi.org/10.1016/j.cell.2014.11.014 (2014).

38. Qian, J. et al. B cell super-enhancers and regulatory clusters recruit AID tumorigenic activity. Cell 159, 1524-1537, https://doi. org/10.1016/j.cell.2014.11.013 (2014).

39. Santos, J. M., Braikia, F. Z., Oudinet, C., Dauba, A. \& Khamlichi, A. A. Two modes of cis-activation of switch transcription by the IgH superenhancer. Proceedings of the National Academy of Sciences of the United States of America, https://doi.org/10.1073/ pnas.1902250116 (2019).

40. Santos, J. M. et al. Duplication of a germline promoter downstream of the $\mathrm{IgH} 3^{\prime}$ regulatory region impairs class switch recombination. Scientific reports 8, 9164, https://doi.org/10.1038/s41598-018-27448-4 (2018).

\section{Acknowledgements}

We thank Fred Alt for advice, Isabelle Robert (Reina San Martin's lab) and Kefei Yu for their help in setting up CH12 transfection conditions, the IPBS animal facility and the Imaging Core Facility TRI-IPBS for their excellent work. Tri-IPBS has the financial support of ITMO Cancer Aviesan within the framework of Cancer Plan. This work was supported by the Agence Nationale de la Recherche [ANR-16-CE12-0017], the Institut National du Cancer [INCA_9363, PLBIO15-134], and the Fondation ARC pour la Recherche sur le Cancer [PJA 20141201647]. CO is a fellow of the Ministry of Higher education \& Research, and recipient of a fellowship from la Fondation pour la Recherche Médicale. LS was recipient of an ERASMUS fellowship.

\section{Author contributions}

J.M.S. performed experiments. C.O., A.D. and L.S. contributed to experiments. A.D. handled the mouse lines. A.A.K. designed the project. J.M.S. and A.A.K. wrote the manuscript. 


\section{Competing interests}

The authors declare no competing interests.

\section{Additional information}

Supplementary information is available for this paper at https://doi.org/10.1038/s41598-019-54929-x.

Correspondence and requests for materials should be addressed to A.A.K.

Reprints and permissions information is available at www.nature.com/reprints.

Publisher's note Springer Nature remains neutral with regard to jurisdictional claims in published maps and institutional affiliations.

(c) (i) Open Access This article is licensed under a Creative Commons Attribution 4.0 International License, which permits use, sharing, adaptation, distribution and reproduction in any medium or format, as long as you give appropriate credit to the original author(s) and the source, provide a link to the Creative Commons license, and indicate if changes were made. The images or other third party material in this article are included in the article's Creative Commons license, unless indicated otherwise in a credit line to the material. If material is not included in the article's Creative Commons license and your intended use is not permitted by statutory regulation or exceeds the permitted use, you will need to obtain permission directly from the copyright holder. To view a copy of this license, visit http://creativecommons.org/licenses/by/4.0/.

(C) The Author(s) 2019 